\title{
SYMBOLS, UNITS AND NOMENCLATURE FOR PHYSICAL QUANTITIES
}

The Commission for Symbols, Units and Nomenclature of the International Union of Pure and Applied Physics has recently issued a document U.I.P. I I (S.U.N. 65-3) which contains the recommendations approved by successive General Assemblies of the International Union of Pure and Applied Physics concerning the symbols used for physical quantities, recommended mathematical symbols, international symbols for units, and recommended nomenclature. These recommendations are in general agreement with the recommendations of the International Organization for Standardization, the General Conference on Weights and Measures, the International Union of Pure and Applied Chemistry, the International Electrotechnical Commission and the International Commission on Illumination.

Standardization of usage in these fields is obviously very desirable, and therefore the Editors of the Journal of Glaciology propose to adopt the recommendations contained in this document as far as possible beginning with No. 49 of the Journal, i.e. at the beginning of Vol. 7 . The main effect of the changes on the style adopted in the Journal of Glaciology will be as follows:

(a) Recognized symbols will be used wherever possible, unless this would result in two physical quantities in the same paper being represented by the same symbol. Thus for example density will be represented by $\rho$ and not by $\gamma$, other things being equal.

(b) Units, even when abbreviated, will not be followed by a full stop and are unaltered in the plural, thus $7 \mathrm{~cm}$ and not $7 \mathrm{~cm}$. or $7 \mathrm{cms}$.

(c) The following mathematical symbols will be used with the following meaning: $\approx$ approximately equal to $\simeq \simeq$ asymptotically equal to $\propto \propto$ proportional to. $\sim$ will not be used.

(d) Changes from previous usage in the fournal of Glaciology as regards abbreviated units will be as follows: s not sec for seconds, $\mathrm{Hz}$ not c/sec for Hertz (cycles per second), deg not $\mathrm{C}^{\circ}$ for temperature interval in degrees (this does not affect the use of ${ }^{\circ} \mathrm{C}$ for degrees Celsius), h not hr for hour, d for day, Ci not $\mathrm{C}$ for Curie.

It will assist the Editors if authors of papers for the fournal of Glaciology will prepare their papers in accordance with these recommendations. Copies of the document may be obtained directly from the Commission, Secretary: Professor J. de Boer, Instituut voor Theoretische Fysica, Valckenierstraat 65, Amsterdam (C), Netherlands. 